\title{
Planar Lombardi Drawings for Subcubic Graphs
}

\author{
David Eppstein \\ Department of Computer Science, University of California, Irvine, USA
}

\begin{abstract}
We prove that every planar graph with maximum degree three has a planar drawing in which the edges are drawn as circular arcs that meet at equal angles around every vertex. Our construction is based on the Koebe-Andreev-Thurston circle packing theorem, and uses a novel type of Voronoi diagram for circle packings that is invariant under Möbius transformations, defined using three-dimensional hyperbolic geometry. We also use circle packing to construct planar Lombardi drawings of a special class of 4-regular planar graphs, the medial graphs of polyhedral graphs, and we show that not every 4-regular planar graph has a planar Lombardi drawing. We have implemented our algorithm for 3-connected planar cubic graphs.
\end{abstract}

\section{Introduction}

Lombardi drawing is a style of graph drawing, named after artist Mark Lombardi, in which the edges of a graph are drawn as circular arcs and in which every vertex is surrounded by edges that meet at equal angles at the vertex-that is, the drawing has perfect angular resolution [9, 10. Several families of graphs are known to have Lombardi drawings, including regular graphs (under certain restrictions on their factorizations into regular subgraphs) and certain highly symmetric graphs [10]. Lombardi drawings have also been used to draw plane trees with perfect angular resolution in polynomial area, something that would be impossible for straight line drawings [9. All graphs have drawings that relax the constraints of Lombardi drawing to allow polyarcs or unequal angles [3, 8 , but the improved aesthetic quality of true Lombardi drawings makes it of interest to determine more precisely which graphs have such drawings.

Planarity, the avoidance of crossing edges, is of great importance both in Lombardi drawing and in graph drawing more generally. By Fáry's theorem, every planar graph can be drawn planarly with straight line segments for its edges, and therefore it can also be drawn with circular arcs. However, those arcs may not meet at equal angles. Not all planar graphs have planar Lombardi drawings [8, 10, and few positive results on planar Lombardi drawing are known: only trees, Halin graphs (the graphs formed from plane trees by adding a cycle connecting the leaves), outerpaths, and the graphs of symmetric polyhedra have been proven to have planar Lombardi drawings [10, 19].

In this paper we take a major step forward in our knowledge of planar Lombardi drawings, and in the applicability of the Lombardi drawing style, by showing that all planar graphs of maximum degree three have planar Lombardi drawings. The heart of our method applies to 3-connected 3-regular planar 
graphs: we apply the Koebe-Andreev-Thurston circle packing theorem to the dual graph, and then use three-dimensional hyperbolic geometry to construct a novel Möbius-invariant Voronoi diagram of the circle packing, which we show to be a Lombardi drawing of the input. Our implementation of this method produces drawings in which the overall drawing and the individual faces are all approximately circular and in which the spacing of the vertices is locally uniform. We extend these results to graphs that are neither 3-regular nor 3-connected by using bridge-block trees and SPQR trees to decompose the graph into 3connected subgraphs, drawing these subgraphs separately, and using Möbius transformations to glue them together into a single drawing. We also use circle packing in a different way to construct planar Lombardi drawings of a special class of 4-regular planar graphs, the medial graphs of polyhedral graphs. However, as we show, not every 4-regular planar graph has a Lombardi drawing.

For space reasons we have omitted some details from this paper, for which see the longer version of the same paper on arXiv:1206.6142.

\section{Preliminaries}

\subsection{Möbius Transformations}

Let $\mathbb{S}^{2}$ denote the space formed by adding a single point $\infty$ "at infinity" to the Euclidean plane; this space is also known as the one-dimensional complex projective line $\mathbb{P}^{1}(\mathbb{C})$. In $\mathbb{S}^{2}$, straight lines may be interpreted as limiting cases of circles, with infinite radius and containing the point $\infty$. A Möbius transformation [24] is a map from $\mathbb{S}^{2}$ to itself that transforms every circle (or line) into another circle (or line). Using complex-number coordinates, these transformations may be represented as the fractional linear transformations and their conjugates. Every two triangles may be mapped to each other by Möbius transformations.

Möbius transformations may also be defined from inversions. If $O$ is a circle centered at point $o$ with radius $r$, inversion through $O$ maps any point $p$ to another point $q$ on the ray from $o$ through $p$, at distance $r^{2} / d(o, p)$ from $o$. $O$ is fixed by the inversion, the inside of $O$ becomes the outside and vice versa, and $o$ trades places with $\infty$. In the limiting case of a line, inversion becomes reflection across the line. Every Möbius transformation may be represented as a composition of a finite set of inversions.

Möbius transformations are conformal mappings: they preserve the angle of every two incident curves. Since they preserve both circularity and angles, they preserve the property of being a Lombardi drawing.

\subsection{Hyperbolic Geometry}

To understand our drawing algorithm, it will be helpful to understand some qualitative features of hyperbolic geometry, avoiding detailed calculations [2].

In the upper halfspace model of three-dimensional hyperbolic space, hyperbolic space is represented by an open halfspace of three-dimensional Euclidean space, 
but with a non-Euclidean distance metric. The boundary plane of the Euclidean halfspace does not belong to the hyperbolic space but may be thought of as the set of "points at infinity" for the hyperbolic space. It is convenient to add one more point $\infty$ to this boundary plane, so that it becomes a copy of $\mathbb{S}^{2}$. Hyperbolic lines are represented by Euclidean semicircles that meet the boundary plane at right angles, or by Euclidean rays perpendicular to the boundary plane; the vertical rays may be viewed as the limiting case of semicircles with one endpoint at $\infty$. Hyperbolic planes are represented by Euclidean hemispheres that meet the boundary plane at right angles in a circle, or by halfplanes that touch the point $\infty$ and meet the boundary plane perpendicularly in a line.

The symmetries of hyperbolic space may be extended to the plane at infinity, on which they act as Möbius transformations. Every Möbius transformation of $\mathbb{S}^{2}$ corresponds uniquely to a symmetry of hyperbolic space.

\subsection{Circle Packing}

In the form that we need it, the Koebe-Andreev-Thurston circle packing theorem 25] states that the vertices of every maximal planar graph may be represented by circles with disjoint interiors, such that two vertices are adjacent if and only if the corresponding two circles are tangent.

It is not known how to find circle packings in strongly polynomial time, but it is possible to find approximate packings by numerical algorithms that are polynomial in both the number of vertices and the accuracy of approximation [22. We use a numerical relaxation procedure by Collins and Stephenson [4] that repeatedly adjusts the radii of individual circles to reduce the angular defect by which the surrounding circles either fail to surround it completely or surround it by a larger angle than $2 \pi$. As Collins and Stephenson show, this method converges rapidly to a unique solution, the system of radii for a valid packing. Once a close approximation to the radii has been calculated, the positions of the circle centers are not difficult to determine.

The circle packings constructed by the Collins-Stephenson procedure (with equal outer radii) yield unsatisfactory Lombardi drawings, with one vertex placed at $\infty$. To improve our drawings, we find a Möbius transformation of the packing
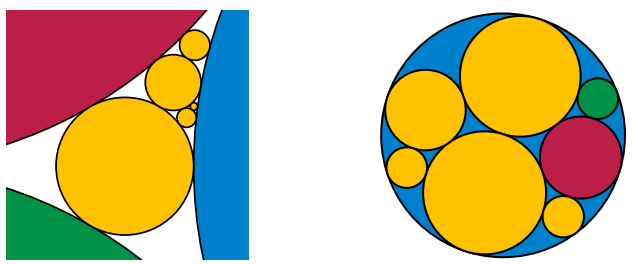

Fig. 1. Left: the central region of a circle packing constructed by the CollinsStephenson procedure; the outer three circles, shown only partially in the figure, have equal radii. Right: a transformed packing with one circle exterior to the others, maximizing the minimum circle radius. 
for which one chosen circle surrounds all the others. Among all transformations fixing the outer circle we choose the one that maximizes the radius of the smallest inner circle (Figure 1). This problem of finding a radius-maximizing Möbius transformation may be expressed (using the connection between Möbius transformations and three-dimensional hyperbolic geometry) as a quasiconvex program, a problem of finding the minimum value of a pointwise maximum of quasiconvex functions. It may be solved either combinatorially in linear time using LP-type optimization algorithms or numerically using local improvement procedures; the theory of quasiconvex programs guarantees that there are no local optima in which the local improvement might get stuck [1,11. Since we are already using a numerical method to find circle packings, our implementation also takes the numerical approach to find the best transformation.

\subsection{Triangle Centers}

A triangle center is a function from Euclidean triangles to Euclidean points such that performing a similarity transformation and then constructing the center gives the same result as constructing the center and then performing the similarity transformation. Hundreds of triangle centers are known, and include many well-known points determined from a triangle, such as its centroid, circumcenter, incenter, and orthocenter [18]. It is convenient, in computing the position of a triangle center, to use barycentric coordinates, weights for which the center is the weighted average of the vertices.

There are two triangle centers that (as an unordered pair of points) are equivariant under Möbius transformations, not just Euclidean similarities. One of these two centers, the first isodynamic point, may be constructed by transforming the given triangle to an equilateral triangle (in such a way that the interiors of the circumcircles of the triangles map to each other), choosing the centroid of the equilateral triangle, and reversing the transformation. The second isodynamic point may be constructed similarly using $\infty$ in place of the centroid.

\section{Cubic Polyhedral Graphs}

We first explain the simplest case of our Lombardi drawing algorithm, in which the planar graph to be drawn is 3-connected and 3-regular. We will later simplify the algorithm, but in terms of three-dimensional hyperbolic geometry, our drawings may be constructed by the following steps:

- Construct the dual graph of the given input graph, a maximal planar graph, and its (unique) planar embedding.

- Apply the Collins-Stephenson procedure to realize the dual maximal planar graph as the intersection graph of a collection of tangent circles $C_{i}$.

- Use quasiconvex programming to find a Möbius transformation of the circles taking them to a configuration in which one circle $C_{0}$ is exterior to all the others, and maximizing the minimum radius of the internal circles. 
- Let the plane on which the circles are packed be the boundary of a threedimensional halfspace model for hyperbolic space. Each circle $C_{i}$ forms the set of points at infinity for a hyperbolic plane $H_{i}$ within this space, which in the halfspace model is represented as a hemisphere. With this model, isometries of hyperbolic space correspond to Möbius transformations of the plane and vice versa.

- Construct the three-dimensional hyperbolic Voronoi diagram of the hyperbolic planes $H_{i}$, a partition of hyperbolic space into cells within which every point is closer to $H_{i}$ (as measured using hyperbolic distance) than to any other one of these planes. The bisector between two hyperbolic planes (the set of points equidistant from both of them) is itself a hyperbolic plane. The cell for $H_{i}$ is a hyperbolic convex polyhedron, the intersection of hyperbolic halfspaces bounded by these bisectors.

- Compute the intersection of the Voronoi diagram boundaries with the plane at infinity (the original Euclidean plane on which we drew the circle packing). The bisectors of the Voronoi diagram form circular arcs in this plane. Triples of bisectors meet at Voronoi edges, which intersect the plane in vertices at which three circular arcs meet. The Voronoi cell for a hyperplane $H_{i}$ meets the plane at infinity in a two-dimensional region, containing circle $C_{i}$ (or contained in it for $i=0$ ) and bounded by circular arcs and vertices. Therefore, the arcs and vertices form a drawing of the dual graph to the intersection graph of circles, which is our initial 3-regular graph.

To verify that this process produces Lombardi drawings, we must show that the three arcs meeting at each vertex form $120^{\circ}$ angles. For any three mutually tangent circles of the circle packing, there exists a Möbius transformation taking their tangencies to an equilateral triangle. In the transformed packing, the bisectors between the three circles are lines, the axes of symmetry of the equilateral triangle, which meet at $120^{\circ}$ angles. Because Möbius transformations are conformal mappings, the three curves in our un-transformed drawing have the same $120^{\circ}$ angles at the vertex where they meet.

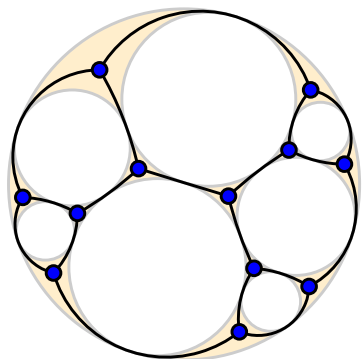

Fig. 2. Lombardi drawing of the Frucht graph derived from the circle packing of Figure 1

Three-dimensional Voronoi diagrams may have quadratic complexity, but we can shortcut this bound by constructing a drawing directly from a circle packing.

Theorem 1. If we are given as input an n-vertex 3-regular 3-connected planar graph, we can produce a planar Lombardi drawing for the graph in time $T+O(n)$, where $T$ denotes the time needed to find a circle packing dual to the given graph.

Proof: Once a circle packing is constructed, the phase of the algorithm that computes a Möbius transformation maximizing the minimum circle radius can be performed in linear time [1]. Each vertex of the drawing lies within the cusp 
formed by three mutually tangent circles. By Möbius invariance, it is the isodynamic point of the triangle formed by the three tangency points of its cusp. We may calculate its position as the weighted average of the three tangency points, using the known barycentric coordinates of the isodynamic point as weights. Each circular arc of the drawing has two vertices as its endpoints and passes through one tangency point, which together determine its location.

\section{Two-Connected Subcubic Graphs}

We next describe how to extend our Lombardi drawing technique to 2-connected graphs. A 2-connected graph may have vertices of degree two or three, but the degree-2 vertices may be suppressed, forming a 3-regular multigraph with the same connectivity. If the multigraph has a planar Lombardi drawing, so does the original graph with the degree- 2 vertices, as the vertices may be restored by subdividing edges without changing the Lombardi property. Therefore, for the most part within this section we assume that the given graph remains 3-regular.

We use a standard tool for decomposing 2-connected graphs, the SPQR tree [5, 6, 16, 17, 20. An SPQR tree for a graph $G$ is a tree structure in which each tree node is associated with a graph $C_{i}$, known as a 3-connected component of $G$. In each 3-connected component, some of the edges are labeled as "virtual", and each edge of the SPQR-tree is labeled by a pair of oriented virtual edges from its two endpoints; each virtual edge of a component is associated in this way with exactly one tree edge. The given graph $G$ may be formed by gluing the components $C_{i}$ together, by identifying pairs of endpoints of virtual edges according to the labeling of the tree edges, and then deleting the virtual edges themselves. The nodes of an SPQR tree have three types: $\mathrm{R}$ nodes, in which the associated graph is 3-connected, S nodes, in which the associated graph is a cycle, and $\mathrm{P}$ nodes, in which the associated graph is a bond graph, a multigraph with two vertices and three or more parallel edges. With the additional constraint that no two $\mathrm{S}$ nodes and no two $\mathrm{P}$ nodes may be adjacent, the SPQR tree is uniquely determined from $G$, and may be constructed in linear time.

The SPQR trees of 3-regular graphs have an additional structure that will be helpful for us:

Lemma 1 (Pootheri [23], Eppstein and Mumford [14]). A 2-connected graph $G$ is 3-regular if and only if each edge in its SPQR tree has exactly one $S$ node as an endpoint, each $S$ node is associated with an even cycle that alternates between virtual and non-virtual edges, each $P$ node is associated with a three-edge bond graph, and each $R$ node is associated with a graph that is itself 3-regular.

Theorem 2. If we are given as input an n-vertex 2-connected planar graph with maximum degree 3, we can produce a planar Lombardi drawing for the graph in time $T+O(n)$, where $T$ denotes the time needed to find and optimally transform a family of circle packings with total cardinality $O(n)$. 

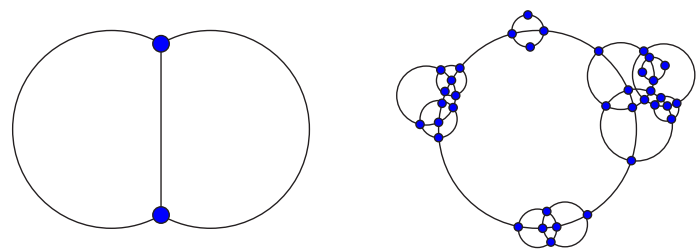

Fig. 3. Left: planar Lombardi drawing for a 3-edge $\mathrm{P}$ node. Right: gluing multiple Lombardi drawings together on an S node with alternating virtual and non-virtual edges (schematic view, not an actual Lombardi drawing).

Proof: Suppress the degree-two vertices of the given graph to produce a 3-regular 2-connected graph, decompose it into an SPQR tree with the additional structure of Lemma 1, and use Theorem 1 to construct a planar Lombardi drawing of the 3 -connected component associated with each $R$ node. In each $\mathrm{S}$ node of the tree, glue the drawings from the adjacent tree nodes together by using inversions centered near their virtual edges to expand the virtual edges, move these edges to the outer face of their drawings, and shrink the rest of the drawings, and then align the circular arcs representing their virtual edges so that they all lie on a common circle (Figure 3, right). Finally, subdivide the edges of the drawing as necessary to restore the suppressed degree-two vertices.

\section{Subcubic Graphs with Bridges}

We are finally ready to describe our algorithm for constructing planar Lombardi drawings for arbitrary subcubic planar graphs, possibly including bridge edges.

Our algorithm begins by deleting all the bridge edges from the graph, and suppressing all degree two vertices, leaving a collection of isolated vertices and 2-connected 3-regular

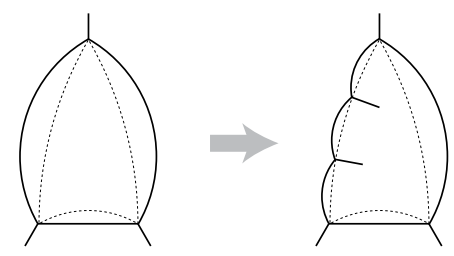

Fig. 4. Modifying a 3-connected Lombardi drawing to attach bridges to subdivision points along an edge subgraphs. It then constructs the SPQR tree of each 2-connected subgraph, forming its 3-connected components, and applies Theorem 1 to construct a planar Lombardi drawing for each $\mathrm{R}$ node in each SPQR tree.

Within each face of each of these drawings, form a sequence of circular arcs forming $30^{\circ}$ angles to the arcs of the face and $60^{\circ}$ angles to each other, forming a smaller inset polygon (Figure 4, left). These arcs cannot intersect each other, as can be seen by considering separately each triangular cusp of the circle packing from which the drawing was constructed. Within each circle of the circle packing (viewing the circle as a disk model for the hyperbolic plane) the new arcs are confined to disjoint halfspaces. For each edge $e$ of one of these drawings that corresponds to a path of suppressed degree-two vertices some of which were 
bridge endpoints, let $k$ be the number of bridge endpoints of $e$. Choose arbitrarily one or the other of the two circular arcs $A$ forming $30^{\circ}$ angles to $e$, and replace $e$ by a sequence of $k+1$ circular arcs, all of which form the same $30^{\circ}$ angle to $A$. These arcs meet each other at $120^{\circ}$ angles, and we may extend a bridge from each of the vertices formed in this way to a new degree-one vertex (Figure 4 right). By a similar construction we unsuppress and attach bridge edges to the suppressed bridge endpoints within the $\mathrm{P}$ nodes and $\mathrm{S}$ nodes of the SPQR tree.

Next, we glue the components within each SPQR tree together, as in Theorem 2, At the same time, for each isolated vertex created by the bridge deletion step, we create a drawing of a claw $K_{1,3}$ consisting of three unit-length line segments meeting at $120^{\circ}$ angles. Additionally, we unsuppress the remaining degree-two vertices of the original graph, by subdividing edges of these drawings. After this step, we have separate drawings for each block (biconnected component) of the original graph, in which each bridge incident to the block has a degree-one vertex at its other end.

Finally, for each two blocks that should be connected by a bridge, we apply an inversion centered on the degree-one endpoint of the bridge, causing the bridge edge to become an infinite ray exterior to the drawing. After this transformation the two blocks may be glued together by making their two copies of the bridge edge lie on the same line. This completes the proof of our main result:

Theorem 3. If we are given as input an n-vertex planar graph with maximum degree 3, we can produce a planar Lombardi drawing for the graph in time $T+$ $O(n)$, where $T$ denotes the time needed to find and optimally transform a family of circle packings with total cardinality $O(n)$.

\section{Four-Regular Graphs}

An alternative form of the circle packing theorem states that every 3 -connected planar graph $G$ and its dual can be simultaneously represented by tangent circles, so that two circles from the two packings are orthogonal if they represent a vertex $v$ of $G$ and a face of $G$ containing $v$, and disjoint otherwise. The packing is unique up to Möbius transformation, and may be found by a numerical procedure similar to the one for circle packing of maximal planar graphs [22].

These dual packings allow us to construct a planar Lombardi drawing of the medial graph of $G$, the 4-regular graph formed by placing a vertex on the midpoint of each edge of $G$ and connecting two of these new vertices by an edge whenever they are the midpoints of consecutive edges on the same face. Each pair of orthogonal circles in the packing and dual packing of $G$ form a lune, which may be bisected by a circular arc forming a $45^{\circ}$ angle with both circles. Two circular arcs meeting at the same point of tangency form right angles or $180^{\circ}$ angles to each other, so the collection of bisecting arcs forms a drawing of the graph with one vertex for each point of tangency and one edge for each pair of orthogonal circles, which is the medial graph of $G$ (Figure 5 , left). As before, this drawing has an interpretation as the intersection of a three-dimensional hyperbolic Voronoi diagram with the plane at infinity, and as before it can be 

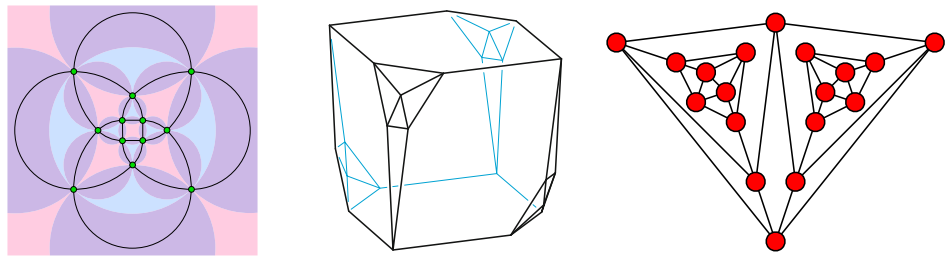

Fig. 5. Left: Dual circle packings for a cube and octahedron, with a Lombardi drawing of their medial graph, the cuboctahedron. Center: A 4-regular polyhedron that is the medial graph of a non-polyhedral graph, described by Dillencourt and Eppstein [13. Right: A 4-regular planar graph with no Lombardi drawing.

extended to certain 2-connected graphs using the SPQR tree. However, this technique does not apply to 3-connected 4-regular planar graphs that are not medial graphs of polyhedral graphs, such as the one shown in Figure 5. center.

The 4-regular 2-connected planar graph in Figure 5, right has no planar Lombardi drawing. We defer the proof to the full version of this paper.

\section{Implementation}

We implemented in Python the algorithm for 3-connected graphs, including the Collins-Stephenson circle packing algorithm and a numerical improvement method for finding optimal Möbius transformations. Our implementation takes as input a text file with one line per vertex; each line lists the identifiers for a vertex and its three neighbors in clockwise order. The output drawing is represented in the SVG vector graphics file format. Figure 6 shows some drawings created by our implementation.

\section{Conclusions}

We have shown that all planar graphs with maximum degree three (and some planar graphs of degree four) have planar Lombardi drawings, greatly extending the classes of graphs for which such drawings are known to exist, and we have implemented our algorithm for the special case of 3-connected 3-regular graphs.

The drawings constructed by our new algorithm have a natural, organic shape, in which the outer face and all the interior faces are approximately circular, resembling soap bubble complexes. If the maximum number of edges per face of the input graph is bounded, then adjacent circles in the circle packing will have radii whose ratio is also bounded, from which it follows that the vertex spacing of the drawing is locally uniform. Our drawings automatically display any global cyclic or dihedral symmetries of the input graph, and may be used even in cases such as the truncated icosahedron which our previous methods, designed for graphs with a high degree of symmetry [10, are incapable of handling. They also display certain local symmetries of the graph, in the sense that, if $G$ is 


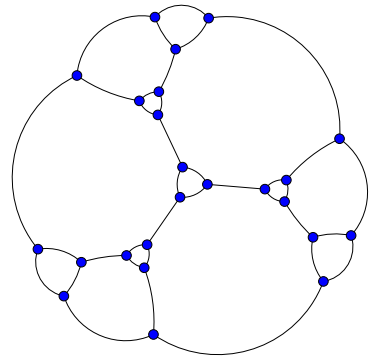

(a) Markström's vertex graph with no 4or 8-cycles 21.

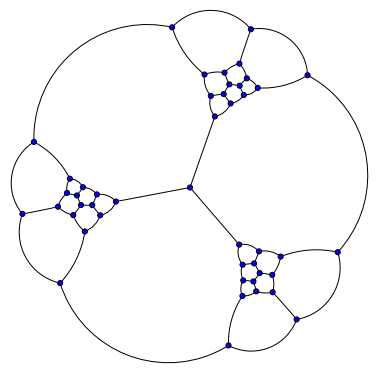

(d) Tutte's 46-vertex nonHamiltonian cubic planar 3-connected graph 26.

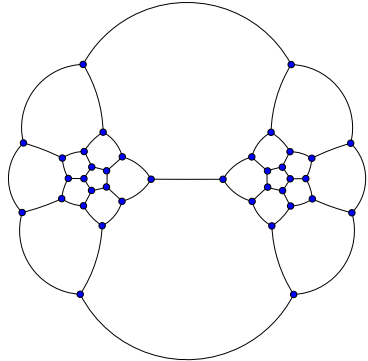

(b) Došlić's 38-vertex graph with girth five and cyclic edge connectivity three 7

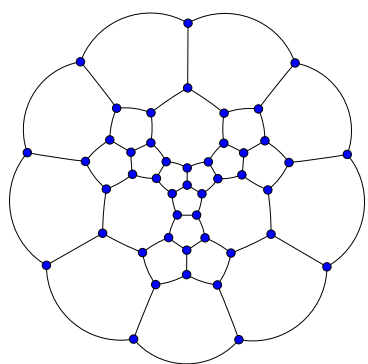

(e) Grinberg's 46-vertex non-Hamiltonian graph with cyclic edge connectivity five 15

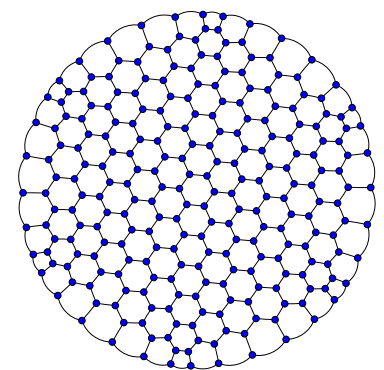

(c) 252-vertex hexagonal mesh

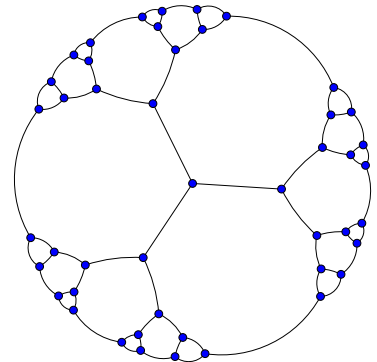

(f) 46-vertex Halin graph formed from a complete ternary free tree

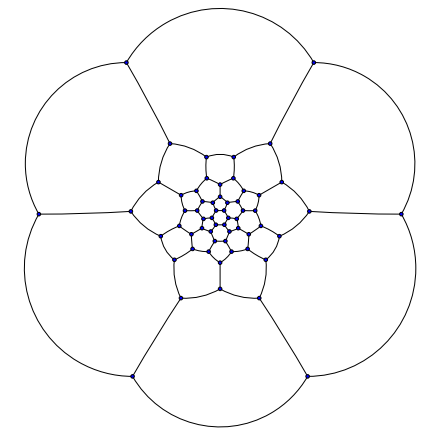

(g) 60-vertex buckyball or truncated icosahedron

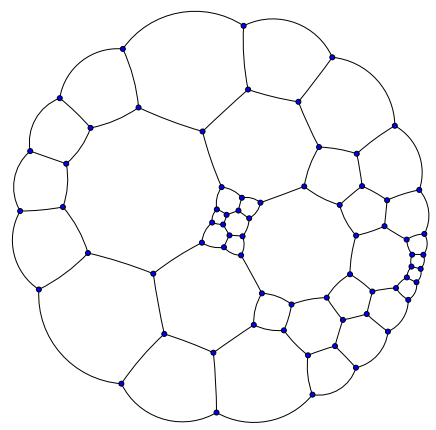

(h) Irregular 69-vertex graph

Fig. 6. Sample drawings from our implementation 
a subgraph of a 3-connected graph that is connected to the rest of the graph by exactly three edges, then every copy of $G$ in one of our drawings can be transformed into every other copy of $G$ by a Möbius transformation.

Possible flaws in this approach, from the graph drawing point of view, are that the area of the drawing (scaled so that the shortest edge has unit length) may be exponential, that the coordinates of the vertices may not be possible to represent precisely (as integers or bounded-degree algebraic numbers) but instead must be approximated numerically, and that the time for computing these drawings depends on the accuracy of this numerical approximation rather than varying strongly polynomially with the input size.

In a followup paper, we extend these results in several directions. In particular, we show that our Lombardi drawing methods for 3-connected and 2-connected planar graphs generate drawings for which it is possible to assign pressures to the faces in such a way that they obey the physical laws governing the static behavior of soap bubbles, and we use this method to characterize the graphs of planar soap bubbles as being exactly the bridgeless 3-regular planar graphs [12. (Our method for graphs with bridges does not produce valid soap bubbles.) In addition, we extend to intersecting disks the Möbius-invariant power diagram defined here for disjoint disks, and we find a distance function in the Euclidean plane for which it is the minimization diagram, giving it an intrinsic definition rather than one relying on hyperbolic geometry.

Although our results greatly extend our knowledge of planar Lombardi drawings, other fundamental questions in this area remain unsolved. For instance, what is the complexity of testing whether a planar graph has a Lombardi drawing? It would also be of interest to determine the maximum number $d$ such that all graphs of maximum degree $d$, or all planar graphs of maximum degree $d$, have Lombardi drawings that are not necessarily planar.

Acknowledgements. This work was supported in part by NSF grants 0830403 and 1217322 and by the Office of Naval Research under grant N00014-08-1-1015.

\section{References}

1. Bern, M., Eppstein, D.: Optimal Möbius Transformations for Information Visualization and Meshing. In: Dehne, F., Sack, J.-R., Tamassia, R. (eds.) WADS 2001. LNCS, vol. 2125, pp. 14-25. Springer, Heidelberg (2001)

2. Cannon, J.W., Floyd, W.J., Kenyon, R., Parry, W.R.: Hyperbolic geometry. In: Levy, S. (ed.) Flavors of Geometry. MSRI Publications, vol. 31, pp. 59-115. Cambridge Univ. Press, Cambridge (1997)

3. Chernobelskiy, R., Cunningham, K.I., Goodrich, M.T., Kobourov, S.G., Trott, L.: Force-Directed Lombardi-Style Graph Drawing. In: van Kreveld, M., Speckmann, B. (eds.) GD 2011. LNCS, vol. 7034, pp. 320-331. Springer, Heidelberg (2012)

4. Collins, C.R., Stephenson, K.: A circle packing algorithm. Comput. Geom. Th. Appl. 25(3), 233-256 (2003)

5. Di Battista, G., Tamassia, R.: Incremental planarity testing. In: 30th Symp. Foundations of Computer Science, pp. 436-441. IEEE (1989) 
6. Di Battista, G., Tamassia, R.: On-Line Graph Algorithms with SPQR-Trees. In: Paterson, M. (ed.) ICALP 1990. LNCS, vol. 443, pp. 598-611. Springer, Heidelberg (1990)

7. Došlić, T.: On some structural properties of fullerene graphs. J. Math. Chem. 31(2), 187-195 (2002)

8. Duncan, C.A., Eppstein, D., Goodrich, M.T., Kobourov, S.G., Löfller, M.: Planar and Poly-arc Lombardi Drawings. In: van Kreveld, M., Speckmann, B. (eds.) GD 2011. LNCS, vol. 7034, pp. 308-319. Springer, Heidelberg (2012)

9. Duncan, C.A., Eppstein, D., Goodrich, M.T., Kobourov, S.G., Nöllenburg, M.: Drawing Trees with Perfect Angular Resolution and Polynomial Area. In: Brandes, U., Cornelsen, S. (eds.) GD 2010. LNCS, vol. 6502, pp. 183-194. Springer, Heidelberg (2011)

10. Duncan, C.A., Eppstein, D., Goodrich, M.T., Kobourov, S.G., Nöllenburg, M.: Lombardi drawings of graphs. J. Graph. Algorithms \& Appl. 16(1), 85-108 (2012)

11. Eppstein, D.: Quasiconvex programming. In: Goodman, J.E., Pach, J., Welzl, E. (eds.) Combinatorial and Computational Geometry. MSRI Publications, vol. 52, pp. 287-331. Cambridge Univ. Press, Cambridge (2005)

12. Eppstein, D.: The graphs of planar soap bubbles (2012), preprint arXiv:1207.3761

13. Eppstein, D., Dillencourt, M.B.: Uninscribable 4-regular polyhedron. Electronic Geometry Models, 2003.08.001 (2003), http://www.eg-models.de/2003.08.001/

14. Eppstein, D., Mumford, E.: Steinitz theorems for orthogonal polyhedra. In: 26th Symp. Comput. Geom., pp. 429-438. ACM (2010)

15. Grinberg, Ė.J.: Plane homogeneous graphs of degree three without Hamiltonian circuits. In: Latvian Math. Yearbook 4, pp. 51-58. Izdat. "Zinatne", Riga (1968)

16. Gutwenger, C., Mutzel, P.: A Linear Time Implementation of SPQR-Trees. In: Marks, J. (ed.) GD 2000. LNCS, vol. 1984, pp. 77-90. Springer, Heidelberg (2001)

17. Hopcroft, J., Tarjan, R.: Dividing a graph into triconnected components. SIAM J. Comput. 2(3), 135-158 (1973)

18. Kimberling, C.: Triangle Centers and Central Triangles. Utilitas Math. (1998)

19. Löffler, M., Nöllenburg, M.: Planar Lombardi drawings of outerpaths (2012) (manuscript)

20. Mac Lane, S.: A structural characterization of planar combinatorial graphs. Duke Math. J. 3(3), 460-472 (1937)

21. Markström, K.: Extremal graphs for some problems on cycles in graphs. Congr. Numerantium 171, 179-192 (2004)

22. Mohar, B.: A polynomial time circle packing algorithm. Discrete Math. 117(1-3), 257-263 (1993)

23. Pootheri, S.K.: Decomposition characterizations of classes of 2-connected graphs. In: 39th Southeast Conf. ACM (2001)

24. Schwerdtfeger, H.: Geometry of Complex Numbers: Circle Geometry, Moebius Transformation, Non-Euclidean Geometry. Dover (1979)

25. Stephenson, K.: Introduction to Circle Packing: The Theory of Discrete Analytic Functions. Cambridge University Press (2005)

26. Tutte, W.T.: On Hamiltonian circuits. J. London Math. Soc. 21(2), 98-101 (1946) 